\title{
EFFECT OF SEVERE PLASTIC DEFORMATION ON MECHANICAL PROPERTIES OF AL6061 / SiC METAL MATRIX COMPOSITES
}

\author{
Siddesha.H.S ${ }^{1}$, Shantharaja.M $\mathbf{M}^{2}$ \\ ${ }^{1}$ Research Scholar, Department of Mechanical Engineering, ACS College of Engineering, Karnataka, India \\ ${ }^{2}$ Assistant Professor, Department of Mechanical Engineering, UVCE, Bangalore University, Karnataka, India
}

\begin{abstract}
The severe plastic deformation process is capable of developing the submicron grain structures in metallic alloys and to improve the mechanical properties. Al6061/SiC composites become one of the most widely used composite materials in the engineering applications. Their light weight, low cost and considerable strength have favored their use in automotive and aerospace industries. In this paper the evaluation of Micro hardness, Ultimate Tensile Strength and ductile properties of severely plastic deformed specimens are discussed. This article describes repetitive corrugation and straightening processes with Cyclic Constrained Groove Pressing parameters such as Wt. \% of Sic, strain rate, Number of passes, Thickness of the specimen and heat treatment process. The results indicated that the percentage of Sic factor has a major influence on the hardness of the composite plate, followed by no. of passes, Heat treatment, plate thickness and strain rate. The optimal conditions for tensile strength are Wt. $\%$ of Sic of $15 \%$, strain rate of $1.5 \mathrm{~mm} / \mathrm{min}$, Number of passes of 7 , specimen thickness of $4 \mathrm{~mm}$ and heat treatment of 3hrs. Experimental results indicate that number of passes and Wt.\% of Sic are most influencing parameters and other parameters are less significant.
\end{abstract}

Key Words: Severe plastic deformation, CCGP, Al/SiC composites, Micro hardness, UTS, Ductility

\section{INTRODUCTION}

The process of severe plastic deformation (SPD) is gaining great interest in material science because it is useful to refine microstructures to the sub micro meter or nanometre levels [1]. In $\mathrm{Al} / \mathrm{Sic}$ composites, it is generally difficult to reduce the grain size below $10 \mu \mathrm{m}$ through the conventional recrystallization process following thermo-mechanical treatments. This difficult arises from the inherent nature of $\mathrm{Al} / \mathrm{Sic}$ composites that the staking fault energy is relatively large so that it is easy for the recovery of dislocation to occur [2]. One of these advantages is a capability of producing large samples that are free from any residual porosity and readily amenable to mechanical testing and forming operations [3]. Among the various SPD techniques proposed most of the methods are intended for processing bulk materials; very few methods like accumulative[4,5] ,repetitive corrugation and straightening(RCS)[8,9] and cyclic constrained groove pressing(CCGP) $[6,7]$ are capable of processing sheet materials. CCGP process was invented recently and it is promising method for producing fine grain sheet material for structural applications. In the CCGP process, a work-piece is repetitively bent and straightened without significantly changing the cross-section geometry of the work-piece, during which large plastic strains are imparted into the materials, which leads to the refinement of microstructure. Although many researchers worked on CCGP but none of them study thoroughly on Metal matrix composite material under severe plastic deformation and CCGP process. In this present study mechanical behavior (Micro hardness, ultimate tensile strength and ductility) were predicted through statistical analysis [10] at different conditions and find the effects of strain rate, number of passes, heat treatment duration, \% of $\mathrm{SiC}$ and plate thickness on prosperities of RCS by using Taguchi L25 orthogonal array [11], to find the influence of various factors and its interaction on the physical behavior of RCS specimens.

\section{EXPERIMENTAL STUDIES}

In the present study, material selected for the composite preparation used $\mathrm{Al} 6061$ alloy. The chemical composition of $\mathrm{Al} 6061$ alloy is $0.8-1.2 \%$ Magnesium, $0.4-0.8 \%$ Silicon, Max $0.7 \%$ Iron, 0.15-0.40\% Copper, Max $0.25 \%$ of Zinc, Max $0.15 \%$ of Titanium, Max $0.15 \%$ of Manganese, 0.04-0.35\% Chromium and balanced \% is Aluminum. Also $\mathrm{SiC}$ of particle size 30 to 50 ? $\mathrm{m}$ used as reinforcement. This was melted at $700^{\circ} \mathrm{C}$ which is slightly more than $30^{\circ} \mathrm{C}$ above the liquiduos temperature. The dispersed used was $\mathrm{SiC}$ particles of size $30-50 \mu \mathrm{m}$, fabricate the specimens, in which a vortex was created in the melt of the matrix alloy through a mechanical stirrer coated with aluminate and rotating at $550 \mathrm{rpm}$. The $\mathrm{SiC}$ particles were preheated to $200^{\circ} \mathrm{C}$ and added to the vortex of liquid melt at a rate of $120 \mathrm{~g} / \mathrm{min}$. A small amount of magnesium, which improves the wet ability of the SiC particles, was added along with the Sic. The composite melt was thoroughly stirred and subsequently degassed by passing nitrogen through at a rate of $2-3-1 / \mathrm{min}$ for 3 to 4 minutes. The composites were then cast in permanent moulds of dimension $20 \mathrm{~mm}$ width, $100 \mathrm{~mm}$ length and $10 \mathrm{~mm}$ thickness. Al alloy composites containing various $\mathrm{SiC}$ contents, namely $5 \%, 10 \%, 15 \%$ and $20 \%$ by weight were fabricated and tested and their properties were compared 
with those of the unreinforced matrix. From the as prepared composites plates the samples were cut with a width of 20 $\mathrm{mm}$ and length $100 \mathrm{~mm}$. The samples are taken in 4 different thicknesses, i.e., $3 \mathrm{~mm}, 4 \mathrm{~mm}, 5 \mathrm{~mm}$ and $6 \mathrm{~mm}$ having same length and width after machining. The $\mathrm{Al}$ alloy MMCs plates with above dimensions were pressed in a corrugated and flat die to produce ultra fine grain structures. These specimens were compressed using four different strain rates i.e. $0.5 \mathrm{~mm}, 1 \mathrm{~mm} / \mathrm{min}, 1.5 \mathrm{~mm} / \mathrm{min}$ and 2 $\mathrm{mm} / \mathrm{min}$ and one to seven passes of compression were considered. The Vickers hardness (Hv) of the test specimens were calculated using Micromet-5101 device, with a load of $200 \mathrm{~g}$ and loading period of 20 seconds. For obtaining reliable results, the measurements of the hardness was performed on all the specimens in three different locations and one among the two nearer value was considered as final hardness value. Tensile test are performed on the specimen with the gauge length of $32 \mathrm{~mm}$ [ASTM E8M standard] width $10 \mathrm{~mm}$ and thickness of $5 \mathrm{~mm}$ specimens machined from the cast composites with the gauge length of the specimen parallel to the longitudinal axis of the castings. Five specimens were tested and the average values of the ultimate tensile strength (UTS) and ductility (in terms of percentage elongation) were measured.

\subsection{Design of Experiments}

Table -1: Factors and their assigned levels.

\begin{tabular}{|l|l|l|l|l|l|}
\hline \multirow{2}{*}{ Factors } & \multicolumn{5}{|c|}{ Levels } \\
\cline { 2 - 7 } & $\mathbf{1}$ & $\mathbf{2}$ & $\mathbf{3}$ & $\mathbf{4}$ & $\mathbf{5}$ \\
\hline SiC (A) & 0 & 5 & 10 & 15 & 20 \\
\hline Strain rate (B) & 0.5 & 1.0 & 1.5 & 2.0 & 2.5 \\
\hline No. of passes (C) & 0 & 1 & 3 & 5 & 7 \\
\hline Thickness (D) & 2 & 3 & 4 & 5 & 6 \\
\hline Heat treatment (E) & 0 & 1 & 3 & 5 & 7 \\
\hline
\end{tabular}

The study was focused on optimization of cyclic constrained groove pressing parameter based on higher strength as well as better ductility. The report was focused on effect of SiC particle, strain rate, number of passes, thickness and heat treatment conditions with five levels. Table 1 shows the five factors and five levels used in these experiments. If five levels were assigned to each of these factors and a factorial experimental design was employed using each of these values, number of permutations would be 3125 experiments. The fractional factorial design reduced the number of experiments to twenty five. Twenty five different experiments were performed using the design parameter combinations in the specified orthogonal array table. Three specimens were fabricated for each of the parameter combinations.

\subsection{Effect of individual factors on Micro hardness}

Taguchi Methods have been successfully applied to Al6061/Sic composite plate subjected to CCGP process parameter optimization in the first step of pressing .From the Experiments it is clear that the parameter reinforcement of $\mathrm{SiC}$ (A) has a highest amount of contribution on response values, Number of passes (C) has the second highest contribution on the response values and Heat treatment (E) the third highest contribution on the response values. Based on the results presented in calculations, reinforcement of Sic is found to be the most influencing process parameter with contribution followed by number of pass, heat treatment, plate thickness and strain rate. Fig.1. shows the main effects plot generated by excels sheet pertaining to ANOVA.

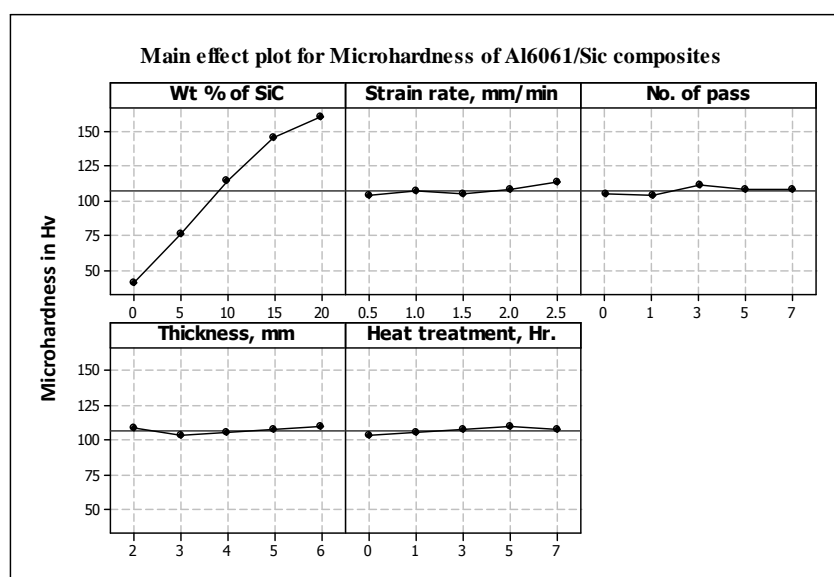

Fig -1: Effect of individual factors on Micro hardness response of $\mathrm{Al} 6061 / \mathrm{SiC}$ composites

\subsection{Effect of individual factors on UTS}

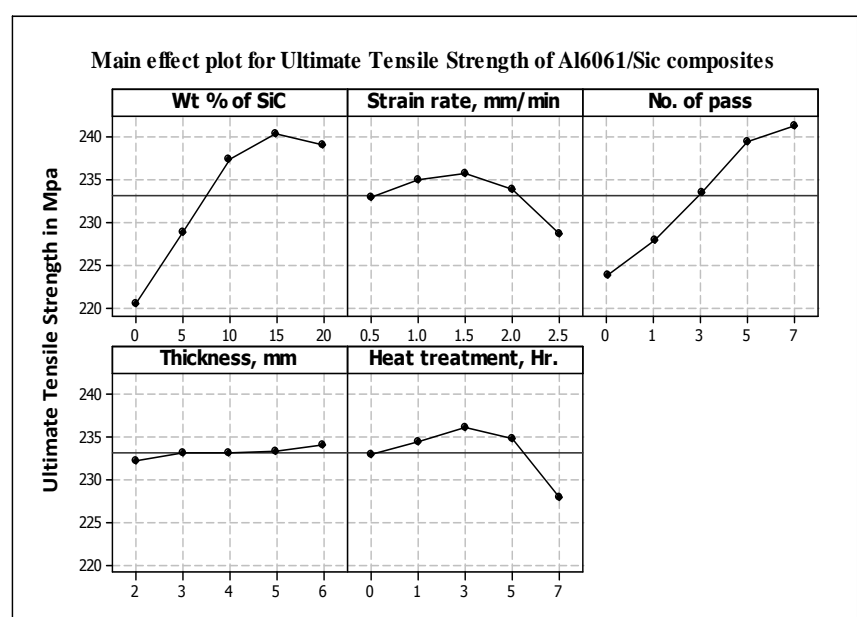

Fig -2: Effect of individual factors on Ultimate Tensile Strength response of $\mathrm{Al} 6061 / \mathrm{SiC}$ composites

Fig.2 shows the mean ultimate tensile strength responses graphically. It is seen that for specified conditions, number of passes, and the wt. \% of $\mathrm{SiC}$ have high significant effects, followed by strain rate. During heat treatment have small effects and thickness of the sample shows no significant effect on UTS. The graphs concluded that number of passes and wt. \% of $\mathrm{SiC}$ have rank 1 and 2 rank respectively and followed by heat treatment (rank 3), strain rate (rank 4) and thickness of the sample (rank 5). Parameter design is an analysis tool that allows the selection of optimum levels for the given experimental variables. In this study, the wt. \% of $\mathrm{SiC}$ and number of passes combinations that produce optimal tensile performance are identified using parameter design. It is easy to find the optimal combination of factor levels necessary to achieve 
peak strength values (maximise UTS) : SiC 15 wt.\%, strain rate $1.5 \mathrm{~mm} / \mathrm{min}$, number of passes 7 , thickness $6 \mathrm{~mm}$ and duration of heat treatment 3 hours.

\subsection{Effect of individual factors on Ductility}

Fig.3 shows the mean ductility responses graphically, Various trial runs under five conditions each of parameters wt.\% of $\mathrm{SiC}$, strain rate, number of passes, thickness and heat treatment. Number of passes (RCS) and thickness of the specimens have no influence on ductility, heat treatment has moderate influence on ductility but wt.\% of $\mathrm{SiC}$ and strain rate has maximum influence on ductility. The addition of $\mathrm{SiC}$ reduces the ductility drastically But similarly strain rate reduces the ductility. Number of passes and heat treatment also though little influence on ductility but they enhance the ductility of the composites. It is also evident that the choice of optimal aging temperature has a greater influence on ductility. The grain boundaries influence on the tensile strength. Higher the grain size lowers the strength and same time lower the grain size also lower the strength.

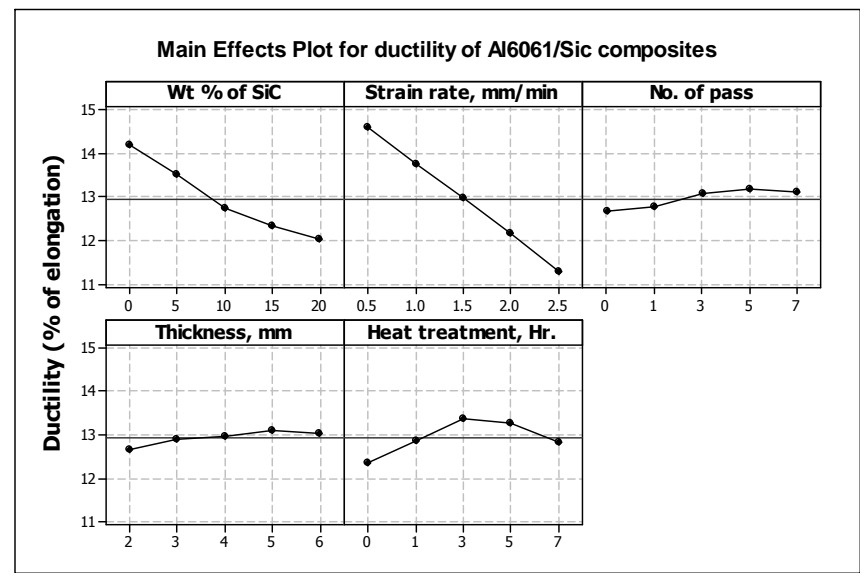

Fig -3: Effect of individual factors on Ductility response of Al6061/SiC composites

\section{CONCLUSIONS}

Taguchi Methods have been successfully applied to AL/Sic composite plate subjected to CCGP process parameter optimization in the first step of pressing At this point it can be deduced that, as far as proper optimization of parameters are needed for maximize hardness, Ulimate Tensile Strength and ductility of Al6061/SiC composites obtained by using the RCS number of passes, RCS strain rate, specimen thickness, heat treatment along with amount of SiC. From the Experiments it is clear that the parameter reinforcement of $\mathrm{SiC}$ (A) has a highest amount of contribution on response values, Number of passes (C) has the second highest contribution on the response values and also The results shows $15 \mathrm{wt}$ \% of $\mathrm{SiC}$ along proper other levels (strain rate of $1.5 \mathrm{~mm} / \mathrm{min}, 7$ number of passes ,specimen thickness of $4 \mathrm{~mm}$ and 3 hours heat treatment)results in reasonable UTS characteristics. In other hand the for ductility $0 \%$ of $\mathrm{SiC}, 0.5$ $\mathrm{mm} / \mathrm{min}$ of strain rate and 3 hour of heat treatment give higher ductility but number of passes and thickens of the specimen have no contribution.

\section{REFERENCES}

[1]. R.Z. Valiev, I.V. Alexandrov, "NanostructuredMaterialsProduced by, Severe Plastic Deformation" Logos, Moscow, 2000.

[2]. T. C. Lowe, R. Z. Valiev (Eds.), "Investigation and Applications of Severe Plastic Deformation" Kluwer, Dodrecht, 2000.

[3]. Z. Horita, $\mathrm{T}$ Fujinami, $\mathrm{M}$ Nemoto, $\mathrm{T}$ G Langdon,"Improvement of mechanical properties for Al alloys using equal-channel angular pressing," Journal of Materials Processing Technology, vol. 117 (2001), pp.288-292

[4]. V.M. Segal, "Materials processing by simple shear", Mater. Sci. Eng. A197 (1995) 157-164.

[5]. N.Tsuji,K.Shiotsuki, H. Utsunomiya, and Y. Saito, in Proceedings of the International Symposiumon"Towards Innovation in Superplasticity-"TransTech.publications.

[6]. Krishnaiah A, Chakkingal U, Venugopal P. Production of ultrafine grain sizes in aluminium sheets by severe plastic deformation using the technique of groove pressing. Scripta Mater 2005;52:122933.

[7]. Z. Horita, M. Furukawa, K. Oh-Ishi, M. Nemoto, T.G. Langdon, in: T. Saki, H.G. Suzuki (Eds.), Proceeding of the fourth International Conference on Recystallization and Related Phenomena, Japan Institute of Metals, Japan, 1999, p. 301.

[8]. J. Gill Sevillano, P. van Houtte, E. Aernoudt, Prog. Mater. Sci. 25 (1980) 69.

[9]. Huang JY, Zhu YT, Jiang H, Lowe TC. Microstructures and dislocation configurations in nanostructured $\mathrm{cu}$ processed BY repetitive corrugation and straightening, Acta Mater, vol.49 (2001), pp. 1497-505.

[9]. S. V. Dobatkin, "Severe Plastic Deformation of Steels: Structure, Properties and Techniques"in Investigation and Applications of Severe Plastic Deformation,Ed. by T. C. Lowe and R. Valiev (Kluwer, Netherlands), 3 (2000) 13-22.

[10]. Cochran, W. G. and Cox, G. M. Experimental designs, 2nd 1968,(Wiley, New York; London).

[11]. Fowlkes, W. Y. and Creveling, C. M., Engineering methods for robust product design, Using Taguchi Methods in technology and product development 1995,(Addison-Wesley Publishing Company). 